\title{
TOMOGRAFÍA DE COHERENCIA ÓPTICA EN LA RETINOPATÍA POR ECLIPSE
}

\section{OPTICAL COHERENCE TOMOGRAPHY IN SOLAR ECLIPSE RETINOPATHY}

\author{
CALVO-GONZÁLEZ C ${ }^{1}$, RECHE-FRUTOS J ${ }^{1}$, SANTOS-BUESO E ${ }^{2}$, DÍAZ-VALLE D ${ }^{2}$, \\ BENÍTEZ-DEL-CASTILLO JM², GARCÍA-SÁNCHEZ J²
}

\begin{abstract}
RESUMEN
Caso clínico: Se describe el caso de un paciente que sufrió pérdida visual aguda tras visualización directa y mantenida de un eclipse solar. El diagnóstico se realiza principalmente mediante tomografía de coherencia óptica.

Discusión: La retinopatía solar es actualmente una causa poco frecuente de pérdida visual. No obstante, se describen algunos casos, especialmente tras la visualización de eclipses de sol. La tomografía de coherencia óptica constituye una prueba diagnóstica adecuada para detectar alteraciones, de forma característica, en las capas más externas de la retina, secundarias a la exposición solar constante.
\end{abstract}

Palabras clave: Retinopatía solar, tomografía coherencia óptica.

\begin{abstract}
Case report: We describe the case of a patient suffering from acute visual loss soon after watching a solar eclipse. Optical coherence tomography was the main diagnostic tool used.

Discussion: Solar retinopathy is now an unusual cause of visual loss, although there are still some cases diagnosed, especially after viewing solar eclipses. Optical coherence tomography is suitable for detecting permanent retinal injuries related to solar exposure, with the outer retinal layers being typically affected(Arch Soc Esp Oftalmol 2006; 81: 297-300).
\end{abstract}

Key words: Solar retinopathy, optical coherence tomography.

\section{INTRODUCCIÓN}

La retinopatía solar aparece documentada desde hace años. Los casos descritos en la actualidad están relacionados con la exposición directa a las radiaciones solares en situaciones bien definidas tales como eclipses solares, enfermedades psiquiá- tricas, rituales religiosos, consumo de drogas alucinógenas, profesionales militares y astrónomos (1).

Se cree que el mecanismo de producción del daño es fotoquímico, afectando fundamentalmente a las capas retinianas más externas. Los síntomas visuales más frecuentes son disminución de la agudeza visual y escotoma central. En la exploración

Recibido: 2/1/06. Aceptado: 28/4/06

Unidad de Superficie e Inflamación Ocular. Servicio de Oftalmología. Hospital Clínico San Carlos. Madrid. España.

1 Licenciado en Medicina.

2 Doctor en Medicina.

Correspondencia:

C. Calvo González

Servicio de Oftalmología. Hospital Clínico San Carlos

Avda. Prof. Martín Lagos, s/n

28040 Madrid

España

E-mail: calvo_glez@yahoo.es 
puede apreciarse una pequeña lesión foveolar de aspecto amarillento $(2,3)$. El diagnóstico se realiza habitualmente mediante la anamnesis y el estudio fundoscópico. La angiografía con fluoresceína no suele mostrar alteraciones. La introducción, recientemente, de la tomografía de coherencia óptica (OCT) ha abierto nuevas posibilidades diagnósticas.

\section{CASO CLÍNICO}

Paciente varón de 30 años de edad, sin antecedentes personales de interés, que acude a urgencias por disminución brusca de visión y escotoma central en su ojo derecho (OD). Refería haber estado mirando directamente y de modo ininterrumpido al sol por el OD durante el eclipse solar del 3 de octubre de 2005 por un período de 3 minutos.

En la exploración, la mejor agudeza visual corregida (AVMC) fue de 20/50 en el OD y 20/25 (Snellen) el ojo izquierdo (OI). El segmento anterior estaba dentro de la normalidad, así como los reflejos pupilares y la presión intraocular. En la funduscopia, el OI era normal y el OD presentaba una leve alteración del epitelio pigmentario retiniano (EPR) así como una lesión amarillenta en el área foveolar (fig. 1). La angiografía con fluoresceína (AFG) se encontraba dentro de la normalidad, así como el campo visual (Octopus- estrategia Top). La rejilla de Amsler no presentaba alteraciones. La exploración con OCT-III manifestó un defecto claro en el segmento externo de los fotorreceptores foveolares, sin otros hallazgos significativos (fig. 2).

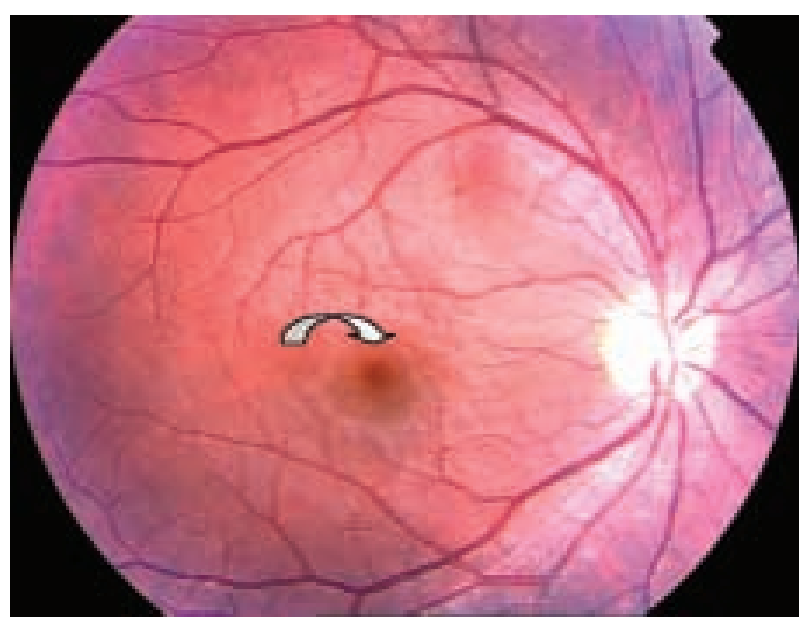

Fig. 1: Imagen funduscópica del OD. Se aprecia una lesión puntiforme amarillenta en área foveolar.

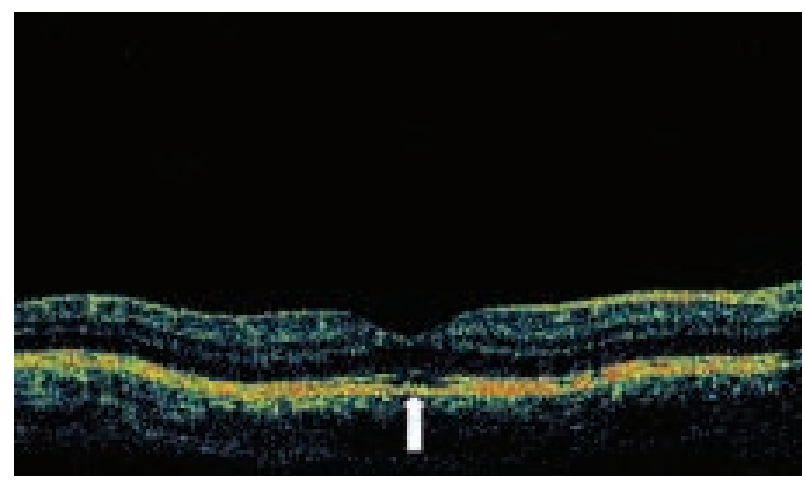

Fig. 2: OCT del OD, donde se aprecia un claro defecto en la capa externa hiperreflectante de los fotorreceptores foveolares.

Se revisó al paciente a los dos meses, repitiendo las pruebas diagnósticas ya realizadas en la primera visita. La AVMC había disminuido en el OD a 20/80, manteniéndose estable en el OI y el paciente refería una clara discromatopsia. El test cromático de Farnsworth 25 realizado no mostraba alteraciones. El OCT presentaba asimismo un defecto del segmento externo de los fotorreceptores foveolares aunque de menor magnitud que el realizado en la primera revisión (el defecto se comparó en el mismo corte de OCT) (fig. 3). Las pruebas restantes realizadas fueron normales.

\section{DISCUSIÓN}

La retinopatía solar se caracteriza por la afectación de las capas más externas de la retina. Se han descrito tres mecanismos que podrían justificar el

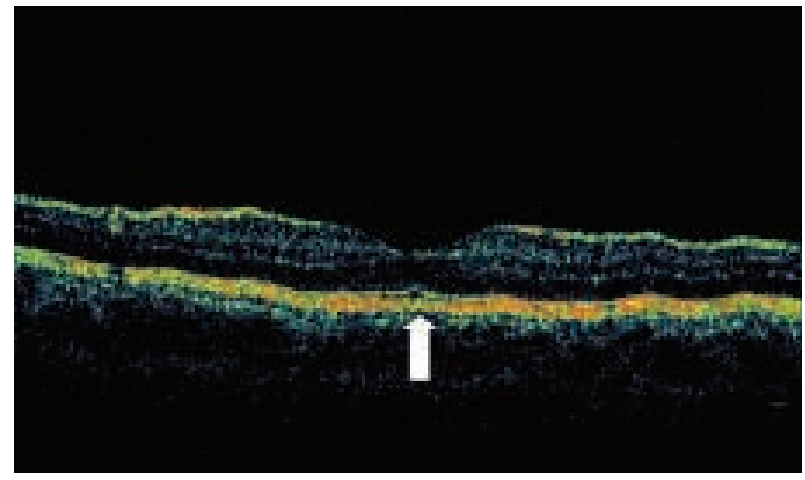

Fig. 3: OCT del mismo ojo dos meses después. Se aprecia un menor defecto en el segmento externo de los fotorreceptores. 
daño retiniano. En el epitelio pigmentario de la retina (EPR) se produce la absorción de un porcentaje importante de radiaciones solares, con un consecuente aumento de la temperatura tisular en las zonas circundantes, produciendo así un daño térmico. Por otro lado, las ondas de alta energía, así como niveles bajos de ultravioleta (UV) A o radiaciones próximas al UV (320-400 nm) son las responsables del daño fotoquímico. En líneas generales, se cree que la afectación retiniana pueda ser consecuencia de la combinación del daño térmico y el fotoquímico (4). Las radiaciones solares producen un daño puntual en los melanosomas apicales del EPR seguido de alteraciones en el segmento más externo de los fotorreceptores foveolares $(1,5)$. El defecto depende directamente de la intensidad, la duración, el espectro de exposición, la dilatación pupilar, la transparencia de medios, la capacidad de enfoque y la refracción, con un riesgo mayor los emétropes o hipermétropes leves, en los que las radiaciones se transmiten directamente a la fóvea (4).

En la exploración funduscópica, es típico observar una lesión amarillenta característica en el área foveolar. Frecuentemente, la AFG es normal aunque, en ocasiones, puede aparecer difusión de contraste en la fase aguda así como defectos ventana en la crónica (2). Las alteraciones descritas por OCTIII demuestran principalmente áreas de excavación en las capas hiperreflectantes del EPR y de los seg- mentos más externos de la capa de fotorreceptores $(1,2,4)$. Algunos autores han descrito por OCT la presencia de un punto hiperreflectante en la cavidad vítrea a unas $70 \mu \mathrm{m}$ de la retina (4).

Los síntomas más frecuentes son disminución de la $\mathrm{AV}$, metamorfopsias, escotoma central, discromatopsia y fotofobia.

En conclusión, el diagnóstico principal de esta entidad se basa fundamentalmente en la anamnesis y la observación funduscópica, dada la escasez de datos que proporciona la AFG. La difusión de la tomografía de coherencia óptica facilitaría el diagnóstico de la retinopatía solar.

\section{BIBLIOGRAFÍA}

1. Kaushik S, Gupta V, Gupta A. Optical coherence tomography findings in solar retinopathy. Ophthalmic Surg Lasers Imaging 2004; 35: 52-55.

2. Jorge R, Costa RA, Quirino LS, Paques MW, Calucci D, Cardillo JA, et al. Optical coherence tomography findings in patients with late solar retinopathy. Am J Ophthalmol 2004; 137: 1139-1143.

3. Yannuzzi LA, Fisher YL, Slakter JS, Krueger A. Solar retinipathy. A photobiologic and geophisical analysis. Retina 1989; 9: 28-43.

4. Codenotti $M$, Patelli $F$, Brancato $R$. OCT findings in patients with retinopathy after watching a solar eclipse. Ophthalmologica 2002; 216: 463-466.

5. Garg SJ, Martidis A, Nelson ML, Sivalingam A. Optical coherence tomography of chronic solar retinopathy. Am J Ophthalmol 2004; 137: 351-354. 